\title{
Healthcare Consultancy System
}

\author{
Prof. Asma Mokashi', Pratik Rughe², Yashashri Malvi2, Neha Ghodekar ${ }^{2}$ \\ ${ }^{1} \mathrm{ME}$ (Computer Engineering), Ph.D. (pursuing), Department of Computer Engineering, SPPU/MMCOE/, Pune, \\ Maharashtra, India \\ ${ }^{2}$ Department of Computer Engineering, SPPU/MMCOE/, Pune, Maharashtra, India
}

\begin{abstract}
Article Info

Volume 7, Issue 3

Page Number: 439-443

Publication Issue :

May-June-2021

Article History

Accepted : 25 May 2021

Published : 31 May 2021

Under the present situation, the healthcare delivery system is prohibitively expensive, inefficient, and unsustainable. Machine Learning (ML) has revolutionized the way businesses and individuals use data to increase system performance. Strategists can work with a range of organized, non - structured, and semi-structured data using machine learning algorithms. This device provides a virtual assistant who can converse with patients in their native language to understand their symptoms, recommend doctors, and monitor health metrics. To process users' complaints and find the closest doctor who can help handle the user's case, the solution relies on natural language processing models and machine learning analytic methodology. A deep bilinear similarity model is also proposed by the framework to boost the generated SQL queries used for predictions and algorithms. BERT and SQLOVA models are used to train the device data collection.

Keywords : Machine Learning (ML), Consultancy System, Web Application Development, Machine Learning Model, Virtual Chat Application, Interactive Chatbot System.
\end{abstract}

\section{INTRODUCTION}

The chat bot technology is automating 2a variety of customer service functions as well as business, institution, and association websites. The user receives a short answer to the questions that are most often asked. For patients, we have proposed a chat bot system. Patients are likely to have a slew of questions about conditions, medications, and other services. Instead of asking a random human, they can use this chat bot system to get a fast answer. A chatbot is an artificial intelligence agent that can engage in communication with a person. The majority of them have a messenger-style gui with a user input and a chatbot output. The chatbot interprets the user's feedback and responds based on what they've just said. It might be a greeting, a topic of conversation, or even a picture. The majority of chatbots balance a user's feedback with a predefined collection of dialogue. For eg, if a user says "Thank you," the chatbot will respond with "You're Welcome." A predefined series of dialogues may be set up to mimic a typical two-person interaction. Problems will happen when a user says something

Copyright: @ the author(s), publisher and licensee Technoscience Academy. This is an open-access article distributed under the terms of the Creative Commons Attribution Non-Commercial License, which permits unrestricted non-commercial use, distribution, and reproduction in any medium, provided the original work is properly cited 
the chatbot doesn't understand. For example, if a user means to say "Thank you," but actually says "Thanks a lot," the chatbot will be confused because it will be searching for "Thank you" feedback to fit with "Welcome." Trying to describe any possible variation of a user saying "Thanks" results in a lot of manual labour. Modern chatbots are more sophisticated, with natural language processing that adapts to user input. They will use APIs to get information from users such as news, weather, time, and so on. They can also use a chatbot interface to process orders and make reservations. Early diagnosis 1of melanoma skin cancer is critical for effective treatment. Melanoma, unlike the other forms of skin cancer, is now well recognised as the most serious because it is much more likely to spread to other areas of the body if not found and treated early. Health machine vision or medical image recognition, which is non-invasive, is becoming highly important in the clinical diagnosis of various diseases. These methods include an automated image processing platform for a precise and fast assessment of the lesion. Collecting dermoscopy image database, preprocessing, segmentation using thresholding, statistical feature extraction using Gray Level Co-occurrence Matrix (GLCM), Asymmetry, Border, Color, Diameter (ABCD), etc., feature selection using Principal component analysis (PCA), calculating total Dermoscopy Score, and then classification using Convocation neural network are all steps involved in this study (CNN). The achieved classification accuracy is 92.1 percent, according to the data.

\section{LITERATURE SURVEY}

Chien-Hao Kao,Chih-Chieh Chen "Model of Multiturn Dialogue in Emotional Chatbot" [1] Chatbots must be able to understand and decipher natural language in nuanced interactions in order to be commercialised. Chatbots are often used to simplify complex activities including shipping new goods to consumers or resolving similar issues, eliminating 2the need for human capital. In medical treatment, text sentiment analysis allows a chatbot to identify and react to a user's emotional state. We merged the multiturn communication and emotion detection models in this analysis 1 to create a chatbot that is better suited for everyday communication rather than computational tasks. As a result, the chatbot can monitor the robot's emotions during a customer encounter. It may also display a variety of emotional responses based on the context of the user's conversation. The dataset is unbalanced because it comes from a television show in which characters often go through intense emotional ups and downs in order to convey the tension of the storey. Sentimentbased identifiers can be used to solve this issue. To make the emotional change feel more incremental rather than abrupt, increase the rating of recurring positive or negative emotions above average. 2The vast majority of existing chatbot training datasets consist of question-answer exchanges, and the responses in everyday contact are bland and cryptic. The quality of a conversation is influenced by a variety of influences. The generative model chatbot uses the Seq2Seq generator from the translation model 1since there are no traditional answers in a conversation. As a result, the study's ability to elicit a variety of responses when faced with a variety of stimuli has been improved. Emotion input to a chatbot is dictated by learning rather than an agent or a rule list, resulting in a more natural response. MMCOE's Computer Engineering Department is based in Pune.

Mubashra Akhtar,Julia Neidhardt." The Potential of Chatbots: Analysis of Chatbot Conversations"[2] . Since the advent of these inventions, 1the idea of using computers to answer questions has existed. The first algorithms aimed at achieving this goal were already in place in the early 1960s. Over the past few years, chatbots have grown in popularity across a 
number of industries. They are viewed as useful tools for enhancing customer satisfaction in business applications. The aim of this paper is to investigate whether customers' chat interactions with a telecommunications company's chatbot can be used to determine a) users' topics of interest and b) user satisfaction. 1To do this, chat messages are interpreted as events and user inputs are analysed using text mining techniques. The results imply that valuable information about users' preferences and satisfaction can be gleaned from their written conversational contributions. If the chatbot is unable to provide the requested answer immediately, the majority of users may leave the conversation after a brief moment. Additionally, most discussions revolve around the same topics. Our results indicate that companies using chatbots should do a thorough analysis of the data they collect 1 in order to get a deeper understanding of their customers' needs. According to our results, they will improve customer satisfaction by offering customised service based on real-time reviews.

Naz Albayrak, Aydeniz O"zdemir and Engin Zeydan. " Yapay Zeka Tabanlı Rehber Robotlara Genel Bir Bakı,s ve O"rnek Bir Rehber Robot Uygulaması An Overview of Artificial Intelligence Based Chatbots and An Example Chatbot Application"[3] As of now, ChatBot can be described as software that communicates with humans through artificial intelligence. These programmes are used to carry out tasks such as effectively responding to users, training them, assisting them in the purchase of products, and providing superior customer service. This article discusses the general operation and fundamental principles of artificial intelligence-based chatbots and related ideas, and also their applications in a variety of companies, including telecommunications, banking, fitness, customer touch centres, and ecommerce. Additionally, the results of an example chatbot for donation programme developed for a telecommunications service provider are presented using the proposed version.

Ramya Ravi. "Intelligent Chatbot for Easy WebAnalytics Insights"[4] Then, in today's fast-paced data-driven world, having trustworthy insights is important for making the best decisions at the right time. Numerous web analytics tools are available that offer performance indicators for online businesses. However, mastering the tools, far less obtaining the knowledge necessary to understand market ramifications, is tedious and time-consuming. I compare the ease of use of two widely used analytics approaches in this article. In light of this, I propose a chatbot operated by Artificial Intelligence Machine Learning (AIML) and fed by raw analytics data that enables bot users to obtain business insights simply by typing in a query. I propose a chatbot throughout this article that enables botusers to easily type in a web analytics query and receive an immediate response. This is to escape the time-consuming process of learning when to use a web analytics tool. The proposed chatbot's data collection is raw analytics data that was generated using AIML. Experiments were conducted to get a better awareness of the tool's capabilities. The tool was evaluated based on its accuracy of reaction, and it performed admirably. Since the chatbot is designed using AIML, the bot-user must obey a script while typing in the query. I'd like to build on my work by fine-tuning and improving the chatbot's intelligence, so that the bot's user is not required to inject the query in a preset pattern.

U R AUVHQLMHY Lu,ODULMD RYL” Artificial intelligence marketing: Chatbots"[5]. Artificial Intelligence is now a technology that allows advertisers to develop extremely customised user interactions, improve the responsiveness of organisations, and solve customer problems. The chatbot is examined as an artificial intelligence platform in marketing in this article, as well as its 
current use and future potential in the abovementioned area. In total, 60 survey respondents were polled about their attitudes, habits, and perceptions while using various communication networks, with a focus on chatbots and their advantages and drawbacks in comparison to other communication channels. The findings revealed that the greatest benefit of using chatbots in marketing services was when delivering easy, fast information, but they also revealed respondents' fear of chatbots providing incorrect information. Chatbots should be considered by businesses, particularly if they face connectivity problems with customers, but also if they want to keep up with the changing lifestyles of their customers.

\section{PROBLEM STATEMENT}

It's tough to get personal access to the hospital and physicians on a regular basis. Approaching hospitals for routine consultation takes time and money. Localized individuals must be able to communicate with medical providers with ease, and can be accomplished using a machine learning technique. The current system is a time-consuming mechanism that is impossible to diagnose in its early stages and signs do not occur in the later stages. Implementation of a method to simplify the classification process in order to diagnose skin cancer early.

\section{PROPOSED SYSTEM}

We are proposing a medical chat and lung cancer detection device. In this method, we are building a Lung Cancer Detection system. To do so, we are providing an image dataset and then pre-processing it by deleting unnecessary data, converting RGB to greyscale, extracting data to verify the image's characteristics, segmenting the image into subparts, and finally classifying it with CNN.

Predict output: Skin Cancer Detect or Not

\section{METHODS AND MATERIAL}

\section{A. System Architecture}

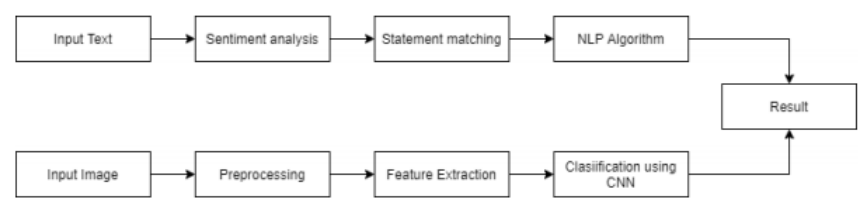

Fig 1.1 Architecture diagram

\section{OBJECTIVE}

The skin cancer identification project's aim is to provide a system for analysing and assessing melanoma risk using dermatological images taken with a normal consumer-grade camera. Personal experience of skin cancer or precancerous lesions. Perform a thorough skin examination on a regular basis, ideally. The skin lesion segmentation step's goal is to locate the skin lesion. Many features used to assess the likelihood of melanoma are extracted from the lesion boundary, so it's critical that this process is done correctly. A texture distinctiveness dependent lesion segmentation is our method for determining the lesion boundary.

\section{CONCLUSION}

It can be inferred that the proposed skin cancer identification method can be applied using a grey level co-occurrence matrix and a help vector machine to quickly classify whether an image is cancerous or not. The suggested system's accuracy rate is $95 \%$. Compared to biopsy, it is a painless and long-lasting procedure. Patients will profit from that further. 


\section{REFERENCES}

[1]. Augello A. Saccone G. Gaglio S. Pilato G., Humorist Bot: Bringing Computational Humour in a Chat-Bot System. Proceedings of the International Conference on "Complex, Intelligent and Software Intensive Systems (CISIS)", 4-7 March 2018, Barcelona, Spain, pp.703- 708.

[2]. Gambino O. Augello A. Caronia A. Pilato G. Pirrone R. Gaglio S., Virtual conversation with a real talking head. Proceedings of the Conference on "Human System Interactions", 25-27 May 2018,Kraow, Poland, pp. 263-268.

[3]. Vojtko J. Kacur J. Rozinaj G., The training of Slovak speech recognition system based on Sphinx 4 for GSM networks. Proceedings of International Symposium "EL, MAR (Electronics in Marine) focused on Mobile Multim

[4]. Sun Microsystems, Developer resources for JAVA technology. [Online] http://java.sun.com (Accessed: 30 Oct. 2018)

[5]. J Abdul Jaleel, SibiSalim, Aswin.R.B," Computer Aided Detection 01 Skin Cancer", International Conference on Circuits, Power and Computing Technologies, 2013. International Research Journal of Engineering and Technology (IRJET) e-ISSN: 2395 -0056 Volume: 04 Issue: $04-\mathrm{Apr}$ -2017 www.irjet.net p-ISSN: 2395-0072 (C) 2017, IRJET - Impact Factor value: 5.181 - ISO 9001:2008 Certified Journal — Page 2881.

[6]. R. Schraer and E. Lawrie, "Coronavirus: scientists brand $5 \mathrm{G}$ claims 'complete rubbish,"' BBC News, 2020. [Online]. Available: https://www.bbc.com/news/52168096.

[Accessed: 05- Apr-2020

[7]. C.NageswaraRao, S.SreehariSastry and K.B.Mahalakshmi "Co-Occurrence Matrix and Its Statistical Features an Approach for Identification Of Phase Transitions Of Mesogens", International Journal of Innovative
Research in Engineering and Technology, Vol. 2, Issue 9, September 2013.

[8]. SantoshAchakanalli G. Sadashivappa ," Statistical Analysis Of Skin Cancer Image -A Case Study “, International Journal of Electronics and Communication Engineering (IJECE), Vol. 3, Issue 3, May 2014.

[9]. "Digital image processing" by jayaraman. Page 244,254-247,270-273. (gray level, median filter).

[10].Algorithm For Image Processing And Computer Vision .Page 142-145.(Thresholding).

\section{Cite this article as :}

Prof. Asma Mokashi, Pratik Rughe, Yashashri Malvi, Neha Ghodekar, "Healthcare Consultancy System", International Journal of Scientific Research in Computer Science, Engineering and Information Technology (IJSRCSEIT), ISSN : 2456-3307, Volume 7 Issue 3, pp. 439-443, May-June 2021. Available at doi : https://doi.org/10.32628/CSEIT217395 Journal URL : https://ijsrcseit.com/CSEIT217395 